\title{
The Robustness of Caenorhabditis elegans Male Mating Behavior Depends on the Distributed Properties of Ray Sensory Neurons and Their Output through Core and Male-Specific Targets
}

\author{
Pamela K. Koo, Xuelin Bian, Amrita L. Sherlekar, Meredith R. Bunkers, and Robyn Lints \\ Department of Biology, Texas A \& M University, College Station, Texas 77843-3258
}

\begin{abstract}
Many evolutionarily significant behaviors, such as mating, involve dynamic interactions with animate targets. This raises the question of what features of neural circuit design are essential to support these complex types of behavior. The Caenorhabditis elegans male uses 18 ray sensilla of the tail to coordinate mate apposition behavior, which facilitates a systematic search of the hermaphrodite surface for the vulva. Precisely how ray neuron types, $A$ and $B$, robustly endow the male with a high degree of spatial and temporal precision is unknown. We show that the appositional postures that drive the search trajectory reflect the complex interplay of ray neuron type-induced motor outputs. Cell-type-specific ablations reveal that the A-neurons are required for all appositional postures. Their activity is instructive because the A-neurons can induce scanning- and turning-like appositional postures when artificially activated with channelrhodopsin (ChR2). B-neurons are essential only for initiation of the behavior in which they enhance male responsiveness to hermaphrodite contact. When artificially activated using ChR2, A- and B-neurons produce different tail ventral curl postures. However, when coactivated, A-neuron posture dominates, limiting B-neuron contributions to initiation or subsequent postures. Significantly, males lacking the majority of rays retain a high degree of postural control, indicating significant functional resilience in the system. Furthermore, eliminating a large number of male-specific ray neuron targets only partially attenuates tail posture control revealing that gender-common cells make an important contribution to the behavior. Thus, robustness may be a crucial feature of circuits underlying complex behaviors, such as mating, even in simple animals.
\end{abstract}

\section{Introduction}

How animals sense the relevant features of their environment and translate this information into appropriate motor responses is perhaps the overarching question in neuroscience. Ultimate and proximate mechanisms underlying mating success and the transmission of heritable information to the next generation is a central concern for evolutionary biology. The nematode worm Caenorhabditis elegans represents a valuable model system for exploring the molecular and cellular underpinnings of these two fundamental questions. Many studies of C. elegans behavior (for

Received Nov. 24, 2010; revised April 1, 2011; accepted April 4, 2011.

Author contributions: R.L. designed research;P.K.K., X.B., A.L.S., M.R.B., and R.L. performed research;P.K.K., X.B., A.L.S., and M.R.B. analyzed data; P.K.K. and R.L. wrote the paper.

This work was supported by National Science Foundation Award 0818595, and M.R.B. by Award EF-0436308. Some nematode strains used in this work were provided by the Caenorhabditis Genetics Center, which is funded by the NIH National Center for Research Resources. We thank Fakhriddin Pirlepesov for suggestions on measuring the tail curvature, Kristin Golla for technical assistance for some of the channelrhodopsin assays, P. Hardin for comments on this manuscript, and L. R. Garcia for helpful experimental advice and manuscript feedback. We also thank the following people for strains and reagents: A. Barrios and S. W. Emmons for A-neuron recCaspase strains; H. Schwartz and H. R. Horvitz for ceh-30(n4111n3714); A. Gottschalk for the mec-4::ChR2(H13R)-YFP plasmid; P. Brockie and A.V. Maricq for the dat-1::/CE plasmid; R. Miller and D.S. Portman for the fs/s 14 strain; and X.Z. S. Xu for the trp- 4 mutant and trp-4:.YFP translational reporter strain.

Correspondence should be addressed to Robyn Lints, Department of Biology, Texas A \& M University, 3258 TAMU, College Station, TX77843-3258.E-mail: rlints@mail.bio.tamu.edu.

DOI:10.1523/JNEUROSCI.6153-10.2011

Copyright $\odot 2011$ the authors $\quad 0270-6474 / 11 / 317497-14 \$ 15.00 / 0$ example, chemotaxis, thermotaxis, and mechanosensation) use hermaphrodites in simple behavioral assays (for review, see Bargmann, 2006; Goodman, 2006; Garrity et al., 2010). These behaviors are mediated by relatively few sensory receptor cells with limited in-built redundancy (Chalfie and Sulston, 1981; Bargmann and Horvitz, 1991; Biron et al., 2008). However, many evolutionarily significant behaviors involve dynamic interactions with animate targets. This raises the question of what features of neural circuit design are essential for supporting these more complex types of behavior. C. elegans male mating behavior consists of a carefully orchestrated sequence of sensorimotor transformations, each triggered by hermaphrodite-derived cues that are sensed by different sensory organs of the male tail (Liu and Sternberg, 1995). Mating begins with initial recognition of a hermaphrodite through contact with the male tail. This induces the male to press his sensilla-studded tail against her surface and perform a systematic search for the vulva. Once the vulva area is sensed, the male begins prodding for the vulval opening with his copulatory spicules. When the spicules breach the opening, they insert fully and the male then transfers sperm. The ray sensilla of the male tail have been implicated in initiating and coordinating mate apposition behavior in which the male maintains tail contact with the hermaphrodite as he travels over her surface in search of the vulva (Liu and Sternberg, 1995). C. elegans hermaphrodites are not 
particularly compliant and may continue to move during mating, making the task of maintaining tail contact all the more challenging (Garcia et al., 2007; Kleemann and Basolo, 2007). Thus, ray sensorimotor circuitry must robustly endow the male with a high degree of spatial and temporal precision, updated on a momentto-moment basis. What are the cellular substrates of the sensorimotor system that simultaneously confer behavioral robustness and sensory precision? The 18 ray sensilla each contain the sensory endings of two morphologically distinct neurons, type A and B (Sulston et al., 1980). Using channelrhodopsin (ChR2), we show that the neurons of all rays share the ability to induce ventral bending of the male tail. This common property would ensure that even fleeting or limited hermaphrodite contact would prompt the male to press his tail against her. A- and B-neurons induce qualitatively different tail postures, and selective ablations of A- and B-populations reveal that the two neuron types do not contribute equally to all appositional postures of the search. Thus, fine control of tail posture during the search stems from dynamic activity of ray neuron types in response to moment-tomoment changes in hermaphrodite surface cues.

\section{Materials and Methods}

Strains

The strains used in this study are as follows: trp-4(sy695)I (Li et al., 2006), cat-2 (e1112)II (Sulston etal., 1975), tph-1 (mg280)II (Sze et al., 2000), mab-5(e2088)III (Kenyon, 1986), lin-39(n1872ts)III (Clark et al., 1993), unc-64(e246)III(Brenner, 1974), pha-1(e2123ts)III (Granato et al., 1994), pkd-2(sy606)IV (Barr et al., 2001), egl-3(ok979)V (Caenorhabditis Genetics Center), him-5(e1490)V, ceh-30(n4111n3714)X (Schwartz and Horvitz, 2007), lin-32(e1926)X (Zhao and Emmons, 1995), bxIs19 (ptrp-4::GFP + ttx-3::GFP), bxIs20 ( ptrp-4::Caspase-3::nz + pgrd-13::cz::Caspase-3 + elt-2::GFP) (Barrios et al., 2008), nIs128 ( $p k d-2:: G F P)$ (Schwartz and Horvitz, 2007), mnIs12 (osm-6::GFP) (Collet et al., 1998), and fsIs14 (ptba-9::YFP + ptba-6::mCherry + psulp-3::GFP) (Hurd et al., 2010). All strains used in this study carried the him-5(e1409) mutation, which generates a high incidence of males (Hodgkin et al., 1979). pha-1(e2123ts) was grown at $15^{\circ} \mathrm{C}$. Strains containing $\operatorname{lin}-39$ (n1872ts) were grown at $25^{\circ} \mathrm{C}$ along with their controls (see Fig. 7). All other strains were maintained at $20^{\circ} \mathrm{C}$.

\section{DNA plasmids}

The following DNA plasmids were used to generate transgenic lines for this study: ppkd-2::ICE (pZL4), ppkd-2::ChR2-YFP (pZL3), pdat-1::ChR2-YFP (pZL12), and ptba-9::ChR2-YFP (pZL13). Except for pgrd-13 (pZL17), all plasmids were generated using the Gateway cloning system (Invitrogen). To make the entry vectors, promoter fragments were PCR amplified from genomic or plasmid DNA templates using gene-specific promoter primers containing attB1 and attB2 sequences. PCR fragments were then cloned into pDONR221 using BP Clonase (Invitrogen) to generate the entry vectors: ppkd-2 ENTRrev (pZL10) or ppkd-2 ENTR (pZL1), pdat-1 ENTR (pZL15), ptrp-4 ENTR (pZL17), and ptba-9 ENTR (pZL14). Promoters were then recombined into the appropriate destination vectors using LR Clonase (Invitrogen). Destination vectors used in this study were $c c d B r e v:: I C E$ (pZL16) and $c c d B:: C h R 2-Y F P$ (pZL5 or pLR167). ccdBrev::ICE was made by digesting pdat-1::ICE (Hills et al., 2004) with Sma1 and Pst1, end-filling, and then inserting the ccdB C.1 cassette (Invitrogen) in the reverse orientation. ccdB::ChR2-YFP (pZL5) was made by digesting mec-4::ChR2(H13R)-YFP (Nagel et al., 2005) with HindIII and BamHI to remove the mec-4 promoter, end-filling, and then inserting the ccdB C.1 cassette. This insertion created a stop codon (TGA) at the cassette/vector junction, which was changed to a glycine codon (GGA) using site-directed mutagenesis to generate pLR167.

The pgrd-13 (pZL17) is a grd-13-promoter-only plasmid and was used to generate the controls strains ( $f k E x 36,37)$ for the A-neuron caspase experiments. pgrd-13 (pZL17) was derived from EM\#317 (Barrios et al., 2008) by digesting with EcoRI and BamHI, end-filling, and then religating. This removes the $c z::$ Caspase- 3 sequence.

\section{Transgenic strains}

Transgenic lines were generated using standard microinjection techniques (Mello and Fire, 1995): fkEx1, [ppkd-2::ChR2-YFP + pha-1(+)]; fkEx9, fkEx41, [ppkd-2::ChR2-YFP + punc-122::GFP]; fkEx12, fkEx13, [ppkd-2::ICE + punc122::GFP]; fkEx30, [punc-122::GFP]; fkEx36, fkEx37, $[p t r p-4+$ pgrd-13 + elt-2::GFP]; fkEx42, fkEx43, [ptba-9::ChR2-YFP + punc122::GFP]; fkIs1, [pdat-1::ChR2-YFP + punc122::GFP]; and fkIs3, [ppkd-2::ChR2-YFP + ttx-3::mCherry].

The above arrays were generated by injecting plasmids at the following concentrations: ppkd-2::ChR2-YFP (20 ng/ $\mu \mathrm{l})$; ppkd-2::ICE (70 ng/ $\mu \mathrm{l})$;

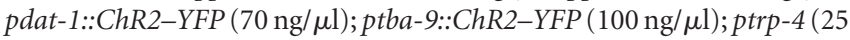

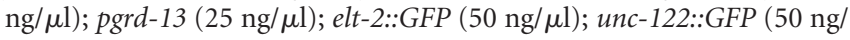
$\mu \mathrm{l})$; and $t \mathrm{tx}-3:: m$ Cherry $(50 \mathrm{ng} / \mu \mathrm{l})$.

Selection of B-neuron pkd-2::ICE and A-neuron caspase males for mating assays

ppkd-2::ICE-mediated killing of B-neurons. The strains used in these experiments carried the transgenic arrays $f k E x 12$ and $f k E x 13$ [ppkd-2::ICE + punc122::GFP] or the control array fkEx30 [punc-122::GFP]. All strains additionally carried a $p k d-2:: G F P$ transgene (nIs128) for visualization of Bneuron cell survival. For mating assays, virgin larval stage (L) 4 males were separated from the stock population (20 males per plate) and allowed to mature overnight. $p$ pkd-2::ICE males lacking all PKD-2::GFP-positive cells were identified using a stereomicroscope equipped with epifluorescence and then selected for mating assays.

Reconstituted caspase-mediated killing of A-neurons. The strains used in these experiments carried the transgenic arrays bxIs20 or bxIs21[ ptrp-4::Caspase-3::nz + pgrd-13::cz::Caspase-3 + elt-2::GFP] (Barrios et al., 2008) or the control arrays fkEx36 or fkEx37 [ptrp-4 ENTR + pgrd-13+ elt-2::GFP]. All strains additionally carried a trp-4::GFP transgene (bxIs19) for visualizing A-neuron cell survival. Cell killing by this caspase system was not as effective as in the $p p k d-2:: I C E$ lines. Animals invariably had surviving A-neurons; therefore, males with the fewest TRP-4::GFP-positive A-neurons were selected for mating assays. Males tested typically lacked A-neurons in at least rays 3,5 , and 8 .

\section{Laser-mediated ray cell rupturing and other ablations}

To ablate specific rays or all rays, L3-L4 larval stage males carrying a ppkd-2::ChR2-YFP or osm-6::GFP reporter were transferred to fresh plates and allowed to mature overnight in the absence of hermaphrodites. For $p p k d-2:: C h R 2-Y F P$ males, the OP50 bacterial lawn was supplemented with $50 \mu \mathrm{M}$ all-trans retinal (ATR), and the number of males per plate was limited to five. Plates were wrapped in aluminum foil because ATR is light sensitive. Individual adult males were mounted on $5 \%$ agarose pads containing $2 \mathrm{~mm} \mathrm{NaN}_{3}$ (anesthetic). To eliminate ray cells by ray process rupturing, the laser was aimed at the ray tips, which contain the processes of the two ray neurons (types A and B) and the ray structural cell, all wrapped in an extension of the multi-nucleate hyp7 syncytium and cuticle. Ray tips were exposed to a laser microbeam until cytoplasm visibly extruded from the ruptured cells. Mock-ablated male controls were mounted for the same period but not laser-treated. All males were allowed to recover from the effects of the anesthetic for at least $5 \mathrm{~h}$ before being subjected to mating assays and ChR2-mediated neuron activation assays. After assays, all males were examined at $600 \times$ magnification on a compound microscope with epifluorescence to assess ray cell integrity and to verify that the rays targeted for killing lacked ChR2YFP- or OSM-6::GFP-positive neurons. In operated animals, the hyp7 syncytium, which covers virtually the entire body and extends to the ray tips, appeared unaffected by the laser treatment as ascertained by examination of the hypodermis with differential interference contrast (DIC) optics, overall animal morphology, and general behavior.

To further verify that the ray process rupturing method was a viable approach to eliminating specific rays, we compared the mating behavior of males lacking $\mathrm{T}$ rays (rays 7-9) as a consequence of ray process rupturing to males lacking these rays attributable to laser ablation of their larval precursors cells. To generate the latter group, L1 animals were mounted on agarose pads containing anesthetic and the ray $7-9$ precursor cells (cells TL and TR) were killed by aiming the laser at their cell bodies (Bargmann and Avery, 1995). Treated and mock-ablated L1 ani- 
Table 1. Samples of raw data used to derive values plotted in Figure 2

\begin{tabular}{lcccr}
\hline Rays remaining & All (mock) & $1-3$ & $5,7,9$ & None \\
\hline Contact response & & & & \\
Max & $100(1 / 1)$ & $100(1 / 1)$ & $69(18 / 26)$ & $22(2 / 9)$ \\
Mean & $60(3 / 5)$ & $52(15 / 29)$ & $46(25 / 54)$ & $6(2 / 31)$ \\
Min & $7(1 / 14)$ & $19(6 / 31)$ & $3(1 / 36)$ & $0(0 / 41)$ \\
Scanning & & & & \\
Max & $451(451 / 1)$ & $565(565 / 1)$ & $307(307 / 1)$ & $10(10 / 1)$ \\
Mean & $68(68 / 1)$ & $74(74 / 1)$ & $35(139 / 4)$ & $2(5 / 2)$ \\
Min & $23(23 / 1)$ & $2(2 / 1)$ & $1(4 / 1)$ & $1(2 / 1)$ \\
Turning & & & & \\
Max & $100(25 / 25)$ & $100(14 / 14)$ & $90(41 / 47)$ & \\
Mean & $71(5 / 7)$ & $67(2 / 3)$ & $20(1 / 5)$ & $0(0 / 1)$ \\
Min & $0(0 / 1)$ & $0(0 / 2)$ & $0(0 / 4)$ & $0(0 / 0)$ \\
\hline
\end{tabular}

Shown are the males that scored the maximum (Max), mean (Mean), and minimum (Min) values for each subbehavior category with raw data in parentheses: contact response (percentage) $=$ (the number of contact responses/total number of tail contacts) $\times 100$; average duration of scanning (in seconds) $=$ (the total time the male had his tail in contact with the hermaphrodite during scanning/total number of contact response events); completed turns $($ percentage $)=($ the number of turns completed/the total number of turning attempts $) \times 100$.

mals were allowed to mature to L4. Males in the population were then transferred to fresh plates and allowed to mature to adults. After mating assays, operated males were examined with DIC optics at $600 \times$ magnification to verify the absence of $T$ rays. In mating assays, males lacking ray cells as a result of laser-mediated ray process rupturing were broadly similar in their behavior to males lacking rays as a result of conventional laser-mediated ablation of ray precursors. In precursor ablated males, 10 of 10 males could turn. In ray process ruptured males, 11 of 11 males could turn.

Laser-mediated ablation of the ray A-neurons was undertaken in a strain carrying the transgene $f_{S I s} 14$ ( ptba-9::YFP + ptba-6::mCherry + psulp-3::GFP) (Hurd et al., 2010), which expresses YFP in the A-neurons and mCherry in the B-neurons. L4 males were isolated from the population and allowed to mature overnight. Adult males were then subjected to laser ablation using standard procedures (Bargmann and Avery, 1995) in which cells are killed by aiming the laser microbeam at the cell body. For each animal, the A-neurons for rays 1-6 were eliminated on both the left and right side of the tail. Mock control animals were mounted with anesthetic for the same duration but not operated on. Males were allowed to recover for $5 \mathrm{~h}$ before being assessed in mating assays. To confirm that A-neurons had been killed in operated animals and that B-neurons were unaffected, males were examined after assaying at $600 \times$ magnification with epifluorescence.

To determine whether CEMs contribute to contact response, the neurons were visualized with $p p k d-2:: G F P$ in adult males, and their cell bodies were laser ablated using standard ablation procedures (Bargmann and Avery, 1995).

The sex-muscle precursor $M$ was eliminated in $p p k d-2:: C h R 2-Y F P$ L1s using standard laser ablation procedures (Bargmann and Avery, 1995). After reaching the L3-L4 stage, males were transferred to fresh ATR plates to mature overnight in the absence of hermaphrodites before being subjected to mating and ChR2 assays.

\section{Mating behavior assays}

Mating assays were performed based on procedures described by Y. Liu et al. (2007). Each assay was digitally recorded using a Carl Zeiss AxioCam HS digital camera and AxioVision software (release 4.7). Trials ended after $15 \mathrm{~min}$ or after the male ejaculated, whichever occurred first. Execution of a specific substep in the behavior was counted using an Excel macro that logs keystrokes and timestamps (Y. Liu et al., 2007). Sample raw data are shown in Tables 1 and 2. Basic measures of mating behavior, such as contact response success, scanning duration, and turn completion rate, were scored as follows. For contact response, male tail contact with the hermaphrodite that resulted in placement of the tail against her surface and backing was scored as contact response. Other types of response that were not counted as a canonical contact response were attempted tail placement without backing, backing without tail placement, or no response. Contact response (percentage $)=($ the number of contact responses/total number of tail contacts $) \times 100$. Average duration of
Table 2. Samples of raw data used to derive values plotted in Figure 3

\begin{tabular}{lccccc}
\hline Treatment & Control (Bs) & - CEMs & $\begin{array}{l}- \text { CEMs, } \\
- \text { HOB }\end{array}$ & Control (As) & As reduced C \\
\hline Contact response & & & & & \\
$\quad$ Max & $100(1 / 1)$ & $100(1 / 1)$ & $100(1 / 1)$ & $100(1 / 1)$ & $94(16 / 17)$ \\
Mean & $78(7 / 9)$ & & $67(2 / 3)$ & $67(2 / 3)$ & $38(10 / 26)$ \\
$\quad$ Min & $7(3 / 41)$ & $50(1 / 2)$ & $0(0 / 5)$ & $0(0 / 5)$ & $0(0 / 22)$ \\
Scanning & & & & & \\
Max & $232(232 / 1)$ & $255(255 / 1)$ & $523(523 / 1)$ & $353(353 / 1)$ & $94(16 / 17)$ \\
Mean & $104(201 / 2)$ & $78(78 / 1)$ & $145(145 / 1)$ & $146(292 / 2)$ & $38(10 / 26)$ \\
Min & $16(94 / 6)$ & $7(7 / 1)$ & $5(5 / 1)$ & $36(36 / 1)$ & $0(0 / 22)$ \\
Turning & & & & & \\
Max & $100(8 / 8)$ & $100(7 / 7)$ & $100(16 / 16)$ & $100(17 / 17)$ & $86(24 / 28)$ \\
Mean & $85(11 / 13)$ & & $83(10 / 12)$ & $88(23 / 26)$ & $24(8 / 34)$ \\
Min & $75(3 / 4)$ & $57(4 / 7)$ & $0(0 / 1)$ & $84(36 / 43)$ & $0(0 / 1)$ \\
\hline
\end{tabular}

See Table 1 legend.

scanning (in seconds) was the total time the male had his tail in contact with the hermaphrodite during scanning/total number of contact response events. Completed turns (percentage) $=$ (the number of turns completed/the total number of turning attempts $) \times 100$. Completed turns include sloppy or good turns followed by resumption of scanning. Turning types were classified as per Loer and Kenyon (1993). All statistical analyses were performed using GraphPad Prism (GraphPad Software).

\section{Channelrhodopsin-2 assays}

All strains used for channelrhodopsin (ChR2) assays were maintained on plates spread with OP50 Escherichia coli containing $50 \mu \mathrm{M}$ ATR and, except during animal transfer or assays, were kept wrapped in foil. Twenty-four hours before assaying, five early L4 males were placed on a plate freshly spread with $50 \mu \mathrm{M}$ ATR plus OP50. Assays were performed on a Carl Zeiss M2 Imager stereomicroscope equipped with epifluorescence. Individual animals were pulsed for $500-700 \mathrm{~ms}$ with blue light (470/40 nm wavelength) while moving forward. Assays were recorded using a Carl Zeiss AxioCam HS camera and AxioVision software. ptba-9::ChR2-YFP ( fkEx42, 43) mosaic males were used to assess the consequence of activating subsets of A-neuron. The identity of neurons activated in these assays was determined after assay at $600 \times$ magnification on a compound microscope equipped with epifluorescence. $p p k d-2:: C h R 2(f k E x 1, f k E x 9)$ lines were essentially non-mosaic, so subsets of ChR2-expressing B-neurons were obtained by specifically rupturing ray cell processes in subsets of rays using a laser microbeam. The absence of ChR2-expressing neurons in laser-treated rays was verified at $600 \times$ magnification as described above. Tail posture was quantified using Axiovision software tools. The frame in which maximum posture response is observed was extracted from the movie. The tail radius of the curvature was measured by drawing a circle that fit the tail curl. To take into account the amount of tail involved in the curl, the radius was multiplied by $c / t c$, where $c$ is the circle circumference $(\pi \times$ diameter), and $t c$ is the length of tail directly in contact with the circle (see Fig. $4 H$ ).

\section{Results}

\section{Background}

The male tail bears nine bilateral pairs of rays (numbered 1 to 9 from anterior to posterior) held together in a cuticular fan (Fig. 1A) (Sulston and Horvitz, 1977; Sulston et al., 1980). All rays are composed of the sensory processes of two ultrastructurally distinct sensory neurons, type A and B, wrapped in the process of a glial-like structural cell. All of these processes are ensheathed in a layer of hypodermis and cuticle. Except for the ray 6 pair, the rays are open at their tip, and the sensory ending of the ray B-neuron is exposed to the environment (Fig. $1 B$ ). Like the hermaphrodite, males lie on their lateral sides and, when not engaged in mating, move backward and forward by propagation of a dorsoventral sinusoidal wave along their body. The cuticular fan in which the rays are embedded drapes down on the ventral side of the tail. Ray 
openings are stereotypically located on the dorsal (d) or ventral (v) surface of the fan or at its margin $(\mathrm{m})$, and these openings are varied along the anteroposterior (A-P) axis (that is, ray $1 \mathrm{~d}, 2 \mathrm{v}, 3 \mathrm{~m}, 4 \mathrm{v}, 5 \mathrm{~d}, 6$ none, 7d, 8v, 9m) (Sulston and Horvitz, 1977; Sulston et al., 1980). Potentially, this design would ensure that, regardless of which part of the fan contacts the hermaphrodite surface during an initial encounter, the B-neuron of at least one ray would directly contact her cuticle. Typically, an initial ray contact with a hermaphrodite induces a contact response: the male bends his posterior ventrally to push his tail down against the hermaphrodite surface so the fan and rays straddle her girth (Fig. 1C) (see Notes). Apposition of the tail against the hermaphrodite puts the hook and post-cloacal sensilla on the tail ventral surface in direct contact with the hermaphrodite cuticle so that these sensilla can sense the vulva when the male passes over it during the search. Initiation and execution of the search are powered by backward locomotion. During the search, if the male tail is far from the hermaphrodite head or tail, he adopts a scanning posture: he keeps the posterior half of his body parallel to the hermaphrodite while maintaining tail contact (Fig. 1D) (see Notes). The male's anterior half may visibly propagate a sinusoidal wave (Barker, 1994; Liu and Sternberg, 1995). However, when the male reaches the hermaphrodite head or tail, he executes a sharp turn that translocates his tail to the other side of the hermaphrodite without losing tail contact. Turning is characterized by a deep ventral bending of the male posterior half and is followed by resumption of scanning along the other side of the hermaphrodite (Fig. 1E) (see Notes). Contact response, scanning, and turning sub-behaviors all involve apposition of the male tail against the hermaphrodite cuticle. We refer collectively to these subbehaviors as mate apposition behavior.

\section{The rays induce tail placement and backing in response to hermaphrodite contact}

All apposition sub-behaviors associated with the vulva search involve tail posture control and backward locomotion. We wanted to know whether the rays control only tail posture, backward locomotion, or both. A straightforward way to address this question is to eliminate all rays and ask what happens to male mating behavior. Liu and Sternberg (1995) eliminated subsets of rays by killing ray progenitor cells using a laser microbeam aimed at the cell body. In addition to eliminating rays, these ablations also eliminated non-ray cell descendents of the same precursors. For example, the PDE and PVD neurons derive from the same precursor as ray 1 (V5.p L/R), and the male-specific hypodermal SET syncitium that sits immediately ventral to rays $1-6$ is derived from the same precursors as these rays (R1-R6) (Sulston and Horvitz, 1977). Conceivably, the absence of these non-ray cells could impact behavior directly or indirectly by distorting tail morphology. Moreover, in conventional laser-mediated cell ablation, there is a risk of collateral damage to neighboring cells because the laser is aimed at the cell body, which is located in ganglia with other cells. Also, because precursors are targeted rather than terminally differentiated cells, there is an opportunity for the system to adjust by developmental compensatory changes that may mask mature neuron function (White et al., 2007). To overcome these limitations, we used an alternative ablation strategy. We eliminated mature ray cells by aiming the laser at ray tips, which contain the processes of the neurons and the glial-like structural cell. This operation caused ray processes to rupture and visibly leak cytoplasm, leading to cell death. To ascertain the effectiveness of this treatment, ablations were performed on transgenic males that express GFP or YFP reporters in ray neurons ( $p p k d-2:: C h R 2-Y F P$, osm-6::GFP). We observed that fluorescence was specifically lost from rays that we targeted with the laser and did not return as would be expected if only photobleaching had occurred or if cells had regenerated their processes. In mating assays, males lacking ray cells as a result of lasermediated ray process rupturing were broadly similar in their behavior to males lacking rays as a result of conventional lasermediated ablation of ray precursors (see Materials and Methods). After a post-operation recovery period of $5 \mathrm{~h}$, individual mockor ray-cell-ruptured males were placed on mating plates with virgin hermaphrodites, and their mating behavior was recorded for the first $15 \mathrm{~min}$ or until they inseminated the hermaphrodite, whichever occurred first (see Materials and Methods). In these assays, we used sluggish hermaphrodites [carrying a mutation in syntaxin gene unc-64 (Saifee et al., 1998)] to reduce hermaphrodite movement and so make quantification of male behavior easier (Y. Liu et al., 2007). Mock-ablated males typically responded on the first or second contact by placing their tail ventral-side down against the hermaphrodite and commencing backing (Fig. $2 A$ ) (see Notes). Tail contact was usually maintained continuously in all apposition sub-behaviors (Fig. $2 B, 2 C$ ), as well as during vulval location, spicule insertion, and sperm transfer. The duration of this contact varied depending on how quickly a male could locate the vulva and inseminate the hermaphrodite. In con- 

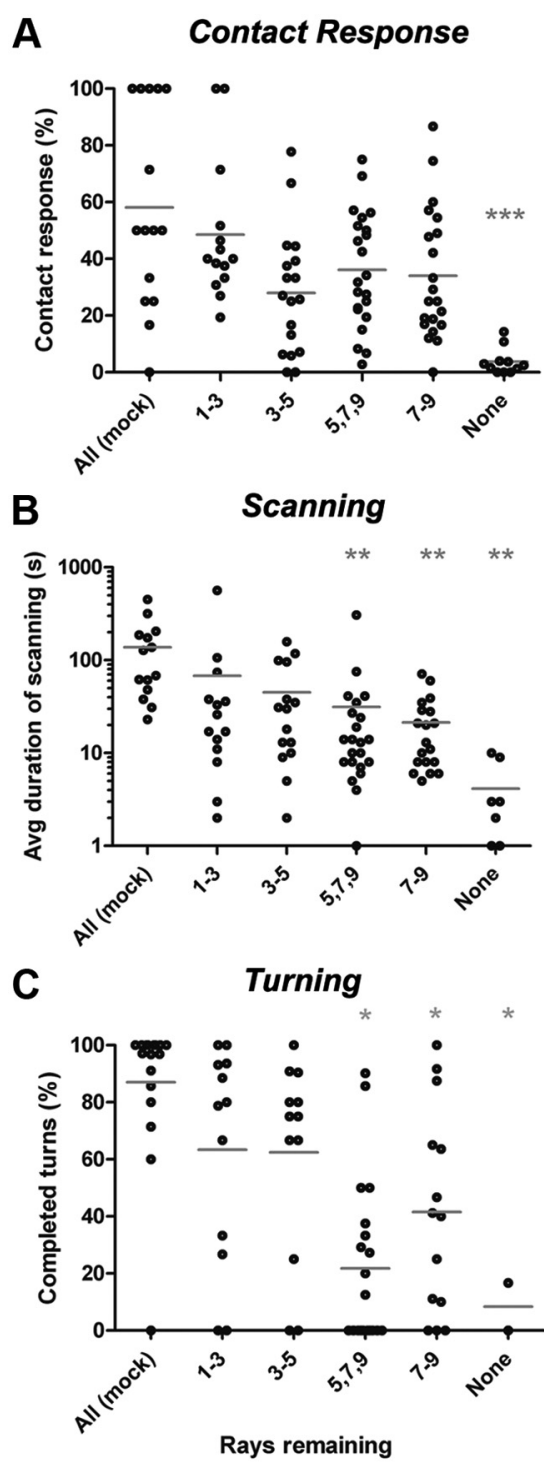

Figure 2. The rays function redundantly in contact response and additively in scanning and turning. All rays or subsets of rays were rendered nonfunctional by rupturing the sensory processes of specific rays using a laser microbeam targeted at the ray tip. After recovery, mock control and operated males were placed with virgin hermaphrodites, and their mating behavior was monitored for $15 \mathrm{~min}$. Each dot corresponds to the performance of a single male with all [All (mock)], none, or specific ray pairs left intact (Rays remaining). The low sample size for males with no rays (None) in $\boldsymbol{B}$ and $\boldsymbol{C}$ is because few males in this category progress as far as scanning or turning. For examples of how graphed values were derived from raw data, see Table 1. Results of nonparametric one-way ANOVA (Kruskal-Wallis test) are shown. Gray bars indicate the mean. Significance is shown as ${ }^{*} p<0.05,{ }^{* *} p<0.01$, and ${ }^{* * *} p<0.001$. For each three-ray-pair combination tested, the orientation of ray tip openings $(\mathrm{d}, \mathrm{v}, \mathrm{or} \mathrm{m})$ are as follows: rays $1-3(d, v, m)$, rays $3-5(m, v, d)$, rays $5,7,9(d, d, m)$, and rays $7-9(d, v, m) . A$, Contact response. Males lacking rays (None) fail to exhibit contact response. All three-ray-pair combinations can respond and at frequencies comparable with males with all rays intact. $\boldsymbol{B}$, Scanning. Males with only rays 5,7 , and 9 or rays $7-9$ intact scanned for significantly shorter periods than males with all rays intact or males with more anterior ray combinations (rays 1-3 or 3-5). $y$-Axis is in log scale. C, Turning. Males with only rays 5,7, and 9 or rays 7-9 intact executed turns significantly less efficiently than males with all rays intact or males with more anterior ray combinations (rays $1-3$ or $3-5$ ).

trast, males entirely lacking functional rays ignored virtually all hermaphrodite contacts made with their tails. A few males did respond at extremely low frequency, but their response was abnormal: they backed in response to hermaphrodite contact but could not place their tails firmly against her surface (Fig. $2 \mathrm{~A}$; for sample raw data, see Table 1) (see Notes). Consequently, these tail contacts lasted only a few seconds (Fig. $2 B$ ). These experiments demonstrate that the rays are responsible for the sensorimotor transformation necessary to mount a contact response. Ray stimulation by hermaphrodite contact induces both tail placement and backward locomotion. Because some contact-induced backing is occasionally observed in males lacking rays, other sensilla may have some capacity to induce a backing response.

\section{The rays function redundantly in contact response and} additively in scanning and turning

Although similar in lineage history and cell composition, the rays are not identical. As described above, the orientation of ray openings alternate along the A-P axis, and ray pairs have a characteristic shape (Sulston and Horvitz, 1977; Sulston et al., 1980). Furthermore, A- and B-type neuron populations can be divided into subtypes based on neurotransmitter fate, and these fates are not exclusively paired with a given ray morphology (Sulston and Horvitz, 1977; Lints et al., 2004). These various features could confer functional specialization among different ray pairs. We therefore asked whether the nine ray pairs contribute equally to all apposition sub-behaviors. To test this, we destroyed subsets of rays (by laser-mediated rupturing of ray cells), leaving different combinations of ray pairs intact. Preliminary experiments suggested that a minimum of three ray pairs were necessary for reproducible response to contact (data not shown) so we based additional behavioral analyses on males with different combinations of three ray pairs intact. We began by testing subsets of rays based on their position along the A-P axis (rays $1-3,3-5$, and 7-9) and then based on shared neurotransmitter fates (for example, rays 5,7 , and 9 , which each contain a dopaminergic A-neuron).

Males with three pairs of functional rays, regardless of the combination, responded to contact at frequencies similar to that of males with all rays intact (Fig. $2 \mathrm{~A}$; Table 1). The slight decrease in their response frequency is possibly attributable to the fact that the surface area for response is reduced when only three functional ray pairs are present. These data argue that the three different $\mathrm{d} / \mathrm{v} / \mathrm{m}$ sets, represented by sequential rays along the A-P axis, are mostly equivalent in their ability to sense contact and to induce contact response.

In contrast to their relatively uniform ability to sense initial hermaphrodite contact, males with only a single intact set of $\mathrm{d} / \mathrm{v} / \mathrm{m}$ opening rays showed marked defects in scanning and turning. For most combinations tested, males lost contact with the hermaphrodite shortly after search initiation (contact response), either during scanning or while executing a turn (Fig. $2 B, C$ ). After loss of contact, these males typically repeated contact response to reinitiate the search and resume their attempts to scan for the vulva. This pattern of initiation, loss of contact, and reinitiation was typically repeated multiple times during the course of a 15 min trial. Although many of the turns that these three-raypair males attempted resulted in loss of contact, a surprising proportion of turns were completed for most three-ray-pair combinations tested. Males with rays $1-3$ and rays $3-5$ intact were significantly better at scanning and turning than males with only posterior rays 7-9. Rays 7 and 9 each contain dopaminergic A-neurons. To test whether dopaminergic rays are generally poor mediators of scanning and turning and thus perhaps specialized for other aspects of male mating behavior, we tested males with only dopaminergic rays, namely rays 5,7 , and 9 . These animals were similar to males with rays $7-9$, suggesting that intact func- 
tion of dopaminergic neurons might contribute little to scanning and turning performance. In contrast, anterior rays, which have cholinergic A-neurons, are more effective controllers of turning and scanning. Thus, differences in the performance of single $\mathrm{d} / \mathrm{v} / \mathrm{m}$ set males during the scanning and turning sub-behaviors could relate to differences in A-neuron neurotransmitter fates.

Overall, our results suggest that all rays have the capacity to sense and induce contact response. There were more marked differences among ray subsets in their ability to mediate scanning and turning, with anterior ray combinations functioning more efficiently than predominantly posterior ray combinations. Scanning and turning efficiency is best when all rays are present. That all combinations tested can mediate these steps suggests that there is functional overlap among the different ray combinations tested. The stochastic nature of their phenotypes suggests that sometimes the activity of neurons in a subset is sufficient to induce the appropriate sensorimotor transformations, but sometimes is not. The results of ray neuron artificial activation, presented below, also support these conclusions. Liu and Sternberg (1995) noted greater difference among rays in their ability to coordinate the ray-controlled steps in mating. For example, they observed that males lacking the three most posterior ray pairs (rays 7-9) could not execute turns. Differences in mating behavior results presented here and from the previous study may be primarily attributable to differences in the methods used to score mating assays. We allowed males an unlimited number of attempts to execute any step of mating within a defined time interval (15 min). For contact response, attempts ranged from 1-54, depending on how many rays were intact (Table 1). In contrast, Liu and Sternberg limited the number of attempts to 10, a much more stringent scoring method that would eliminate males with a tendency to perform below threshold but could potentially execute a step given sufficient opportunities.

\section{Ray A- and B-type neurons have both common and distinct roles in mating}

Each ray contains the dendritic endings of two sensory neurons, type A and B. The B-neuron sensory ending is ciliated and extends to the tip of the ray and so is exposed to the environment in all rays except ray 6, which lacks an opening (Fig. $1 B$ ). The A-neuron sensory ending terminates short of the ray tip and contains a striated rootlet (Sulston and Horvitz, 1977; Sulston et al., 1980). These morphological differences suggest that A- and $\mathrm{B}$-neurons might sense different stimuli during mating. We therefore asked, what role do the A- and B-neuron types within each ray play in the execution of the different apposition subbehaviors? To address this question, we specifically eliminated either A- or B-neurons and assessed the impact on mating. Because laser ablation of neuron processes at the ray tip is not sufficiently accurate to selectively target either the A- or B-neuron process within a ray, we eliminated each neuron type by alternative means: through genetically targeted expression of heterologous cell death genes or by killing cells with conventional laser ablation approaches in which the laser microbeam is aimed at the neuron cell body (Bargmann and Avery, 1995; Hills et al., 2004; Chelur and Chalfie, 2007).

We specifically eliminated B-neurons by driving expression of the human cell death gene ICE (for interleukin-1 $\beta$-converting enzyme) to these cells with the $p k d-2$ promoter ( $p p k d-2)$. This promoter is expressed in the B-type neurons of all rays (except that of ray 6) as well as the male-specific hook HOB neuron and the four CEM neurons of the head (Barr and Sternberg, 1999). The presence of an integrated $p p k d-2:: G F P$ reporter gene (nIs128) in these strain backgrounds made it possible to readily determine the extent of cell death in each animal, and only males in which all PKD-2::GFP-positive ray neurons were eliminated were selected for mating analyses. These B-neuron-deficient males (Fig. 3A, $-\mathrm{CEMs},-\mathrm{Bs},-\mathrm{HOB}$ treatment) exhibited noticeable defects in contact response. Thirty percent of B-neuron-deficient males ignored all hermaphrodite contact during the trial (Fig. 3A; for sample raw data, see Table 2). The remaining $70 \%$ responded to hermaphrodite contact but only after multiple encounters involving tail contact. On average, they required twice as many contacts as control males before responding. Significantly, their eventual response as well as their execution of scanning and turning was indistinguishable from that of control males (Fig. $3 B, C$ ) (see Notes). Their contact response defects could be attributed to the absence of B-neurons specifically because males lacking CEMs (Fig. 3A,-CEMs treatment) have no defects in contact response, and the elimination of the hook, which contains $\mathrm{HOB}$, does not affect contact response (Liu and Sternberg, 1995). Also, their eventual response was not dependent on the activity of surviving B-neurons of the ray 6 pair, because ablation of this ray pair did not alter response frequencies (data not shown). The B-neurons therefore enhance the male's ability to respond to contact but appear dispensable after this step.

To specifically eliminate A-neurons, we used two different approaches: genetically targeted expression of reconstituted human Caspase-3 (recCaspase) to the A-neurons and lasermediated cell ablation of A-neurons. In the recCaspase strains, the $\mathrm{N}$ - and C-terminal halves of human Caspase-3 are expressed from different promoters that overlap only in the A-neurons (Barrios et al., 2008), so it is only in these cells that a fully reconstituted, functional Caspase-3 is formed. A-neuron cell death, indicated by a reduction in TRP-4::GFP-positive A-neurons, was less extensive than for B-neurons in the ppkd-2::ICE strains. Typically, only $30 \%$ of A-neurons were eliminated (most often, those of rays 3,5 , and 8 ). The laser ablation method enabled us to eliminate a greater proportion of the A-neuron population. Laser ablations were performed in a strain carrying the transgenic array $f_{S I}$ Is 14 , which expresses YFP in the A-neurons and mCherry in the B-neurons (Hurd et al., 2010). The use of this two-color system to distinguish A- versus B-neurons enabled us to verify that Aneuron ablation did not inadvertently cause collateral damage to the B-neurons, which are located in the same ganglia. We could reliably eliminate $60 \%$ of the A-neurons (A-neurons of rays 1-6) using this approach. Both the recCaspase transgenic males (Fig. 3, As reduced C treatment) and the laser-ablated males (Fig. 3, As reduced $\mathrm{L}$ treatment) exhibited defects in contact response, scanning, and turning (see Notes). In general, the defects were more severe in the "As reduced L" males, consistent with these males having fewer functional A-neurons. As reduced L males frequently lost contact during scanning (Fig. 3B). Also, these males often failed to complete turns or did not turn when they reached the end of the hermaphrodite, scanning past the end and losing contact (Fig. 3C). Together, these A-neuron-killing experiments indicate that the A-neurons are critical for all mate apposition sub-behaviors.

In contrast to the B-neuron-deficient phenotype, the A-neuron defects were stochastic: the same male would sometimes execute a given step as per wild type and sometimes abnormally. The stochastic nature of this behavioral deficit is reminiscent of males with only subsets of rays and is possibly the consequence of incomplete A-neuron population elimination. That is, in the absence of some A-neurons, remaining A-neurons are sometimes sufficient for sensorimotor control but sometimes are not. Sto- 
A

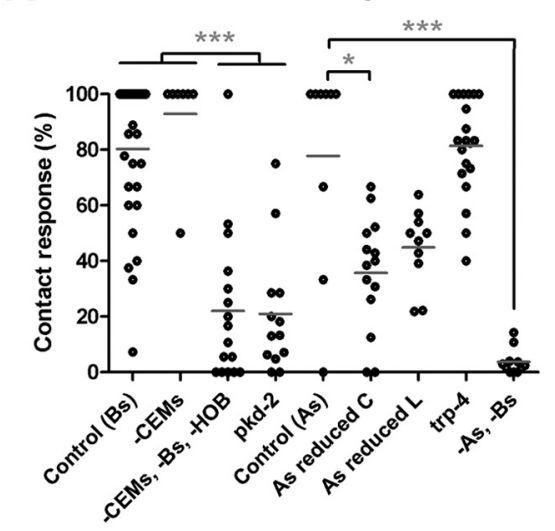

B

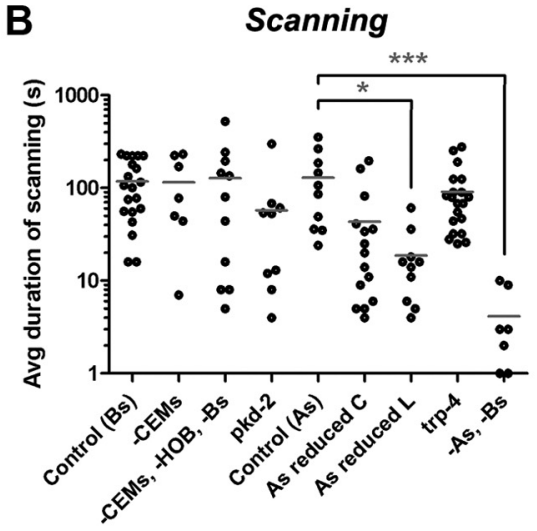

C

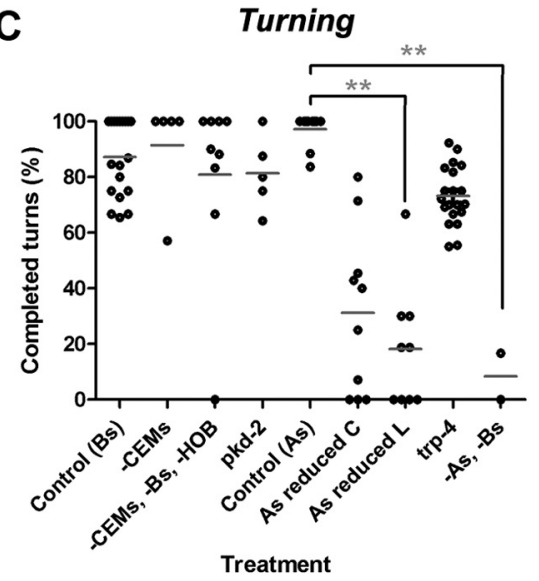

Figure 3. A- and B-type ray neurons have overlapping and distinct functions in control of mate apposition behavior. Males specifically lacking either A- or B-type ray neurons were assessed for their ability to execute $\boldsymbol{A}$, Contact response. $\boldsymbol{B}$, Scanning. $\boldsymbol{C}$, Turning. Control (Bs) correspond to $p p k d-2:: G F P$ ( $f k E x 30$ ) transgenic males that do not carry the ppkd-2::/CE transgene. "As reduced C" correspond to the transgenic recCaspase males, and "As reduced L" correspond to males in which A-neurons of rays 1- 6 have been ablated with the laser. Control (As) are trp-4::GFP ( fkEx36,37) transgenic males that do not carry the recCaspase transgenes and the $f s / s 14$ mock controls for the A-neuron laser ablation experiments. Males lacking both $A$ - and B-neurons ( $-A s,-B s$ ) were generated by eliminating both neuron types through laser-mediated ray cell process rupturing. pkd-2 corresponds to pkd-2(sy606) null mutants males and trp-4 to trp-4(sy695) null mutant males. In $\boldsymbol{B}$ and $\boldsymbol{C}$, the $n$ of "-As, $-B s$ " males is low because males lacking both ray neurons rarely respond to contact and so rarely progress to scanning or turning. For examples of how graphed values were derived from raw data, see Table 2. Results of nonparametric one-way ANOVA (Kruskal-Wallis test) are shown. Gray bars indicate mean. Significance is shown as ${ }^{*} p<0.05,{ }^{* *} p<0.01$, and ${ }^{* *} p<0.001$. chastic behavior also suggests that there is some functional overlap among A-neurons of different rays. Overall, these cell-typespecific ablation studies suggest that both A- and B-neurons are required for contact response, whereas turning and scanning depend primarily on A-neuron function.

\section{TRP channels contribute differentially to A- and B-neuron sensory function}

A question of obvious interest is, what types of sensory receptor molecules do A- and B-neurons use to register hermaphrodite contact? The molecular identity of these receptors could provide insight into the modality of the ray neurons. Transient receptor potential (TRP) channels have been implicated in mechanosensory transduction in a number of systems, although in many instances, it has been difficult to determine whether they directly sense mechanical stimuli or function downstream in signal amplification (Christensen and Corey, 2007). The ray neurons express two TRP channel genes, $p k d-2$ and $\operatorname{trp}-4$. PKD-2 is the $C$. elegans polycystin-2 (PC-2/TRPP2) channel ortholog (Barr and Sternberg, 1999; Knobel et al., 2008). It is specifically expressed in the CEMs, HOB, and B-neurons, in which it localizes to the endoplasmic reticulum and to cilia structures in the neuron sensory endings (Barr and Sternberg, 1999). As reported previously, we find that $p k d-2$ null mutant males have contact response defects (Barr and Sternberg, 1999; Barr et al., 2001; Miller and Portman, 2010). In fact, $p k d-2$ males are behaviorally similar to $p p k d-2:: I C E$ males (Fig. 3). Thus, pkd-2 loss of function results in essentially the same functional outcome as elimination of the B-neurons. These data therefore support the hypothesis that PKD-2 may be part of the B-neuron sensory receptor complex or is at least essential for B-neuron function. In addition, our cell ablation data point to the B-neurons as the site of action for PKD-2 in mediating contact response because defects in this sub-behavior are only observed in the treatment in which B-neurons are absent (Fig. 3).

C. elegans trp-4 encodes an TRPN-type channel related to Drosophila and zebrafish NompC (Walker et al., 2000; Sidi et al., 2003). YFP-tagged TRP-4 fusion protein is expressed in the A-neurons of all rays (except that of ray 4) in which it localizes primarily to the sensory endings (data not shown) (Li et al., 2006). TRP-4 is also expressed in 10 sex-common (core) neurons (Li et al., 2006) in which it functions as the pore-forming subunit of a mechanosensory-gated channel (Kang et al., 2010). To determine whether TRP-4 might have a similar function in the ray A-neurons, we assessed the mating behavior of trp-4 mutant males. A prediction of our A-neuron ablation data is that, if TRP-4 is an essential component of the A-neuron sensory receptor apparatus, trp-4 mutant males should behave like A-neuronablated males and have significant defects in all apposition sub-behaviors. Surprisingly, however, we find that trp-4 null mutants display only minor impairments in apposition behavior. In all sub-behaviors, trp-4 males made more errors than control males, but their error rate was not significant (Fig. 3). Both $\operatorname{trp}-4$ males and hermaphrodites are hyperactive and exhibit exaggerated body bends during locomotion attributable to the absence of TRP-4 in core cells (Li et al., 2006). To determine whether male hyperactivity might contribute to the error frequency of $\operatorname{trp}-4$ males, we compared the speed with which control and $\operatorname{trp}-4$ males scanned hermaphrodites. We observed that $\operatorname{trp}-4$ males scan consistently faster than controls (mean scanning rate for controls, $7.7 \mathrm{~s}$ /hermaphrodite non-vulva side; trp-4 males, 5.4 s/hermaphrodite non-vulva side; $p<0.05)$. Thus, hyperactivity during scanning and turning could account for the higher error rate of $\operatorname{trp}-4$ males. Together, these data suggest that, in contrast 
to its role in the core neurons, TRP-4 is not an essential component of the A-neuron sensory machinery. It is possible that trp-4 is involved in sensory function but that other factors can compensate for its loss of activity. Theoretically, PKD-2 activity in the B-neurons could be responsible for such compensation, but this scenario is unlikely given our cell ablation data, which show that the B-neurons cannot compensate for A-neuron dysfunction. A more likely possibility is that an unknown receptor component operating in the A-neurons may be compensating for the absence of TRP-4 function in these cells. Alternatively, an entirely different class of sensory channel may be activated by stimuli, such as DEG/rENaC or Piezo orthologs (Chelur et al., 2002; Coste et al., 2010), with the ray-expressed TRP channels perhaps acting to modulate the gain of the sensory signal (Christensen and Corey, 2007).

\section{A- and B-neuron populations induce distinct tail-curl postures}

To better understand the relative contribution of $\mathrm{A}$ - and B-neuron populations to posture control and backing, we artificially activated each cell type, in the absence of a hermaphrodite stimulus, using the light-inducible cation channel channelrhodopsin (ChR2) (Nagel et al., 2005). To target expression of this channel to B-neurons, we placed ChR2 tagged with YFP (ChR2$Y F P$ ) under control of the $p k d-2$ promoter. To drive expression of ChR2-YFP to A-neurons, we placed the channel under the control of either the dat-1 promoter ( $p$ dat-1), which is expressed in dopaminergic A-neurons (located in rays 5, 7, and 9), or the tba-9 promoter ( $p t b a-9)$, which is expressed in the A-neurons of rays 3 , $4,6,7$, and 9 and occasionally in those of rays 1 and 2 (Hurd et al., 2010). To activate the channel, we exposed virgin, adult transgenic males to a $500 \mathrm{~ms}$ pulse of blue light and observed their response. ChR2-mediated channel activation of either A- or B-neurons caused males to curl their tails ventrally (Figs. 4, 5). Although all promoters used in these experiments are additionally expressed in some non-ray neurons, control experiments verified that the tail-curling response correlated with activation of male ray neurons (Fig. 5A, C). We derived a tail-curve value for each male tested using a formula based on the tail-curl radius of curvature $(r)$ and length of the tail involved in the curl $(t c)$ (Figs. $4 H, 5)$ (see Materials and Methods). For both A- and B-neuron activation, tail curling was accompanied by limited backing in $50 \%$ of B-neuron-activated males $(n=40)$ and in $20 \%$ of A-neuronactivated males $(n=37)$ that express ptba-9::ChR2-YFP. In some treatment groups in these experiments (see controls in Figs. $4 A$, $5 A, C)$, animals did not curl their tails or alter their forward locomotion in response to the light pulse. For these nonresponders, we measured tail curvature during forward locomotion at the time interval in which a response is normally observed after a light pulse.

The tail-curl postures induced by ChR2-mediated A- and B-neuron activation were not identical. They differed primarily in the openness of the curl and the degree of relaxation in the region between the midbody and the tail-curl flexure point (Fig. 4). B-neuron activation in the majority of males induced a tight circinate curl (tail-curve values ranging from 10 to 20) (Fig. $4 B, C)$ (see Notes). The body region anterior to the curl typically bowed ventrally as if dorsal muscles were contracted and ventral muscles relaxed. Activation of various three B-neuron-pair combinations produced similar postures to that generated by eight B-neuron pairs (Figs. $4 B, C, 5 B$ ), although the frequency of circinate posture was somewhat decreased (from 58\% for eight B-neuron pairs to $12-27 \%$ for three B-neuron pairs). Thus,

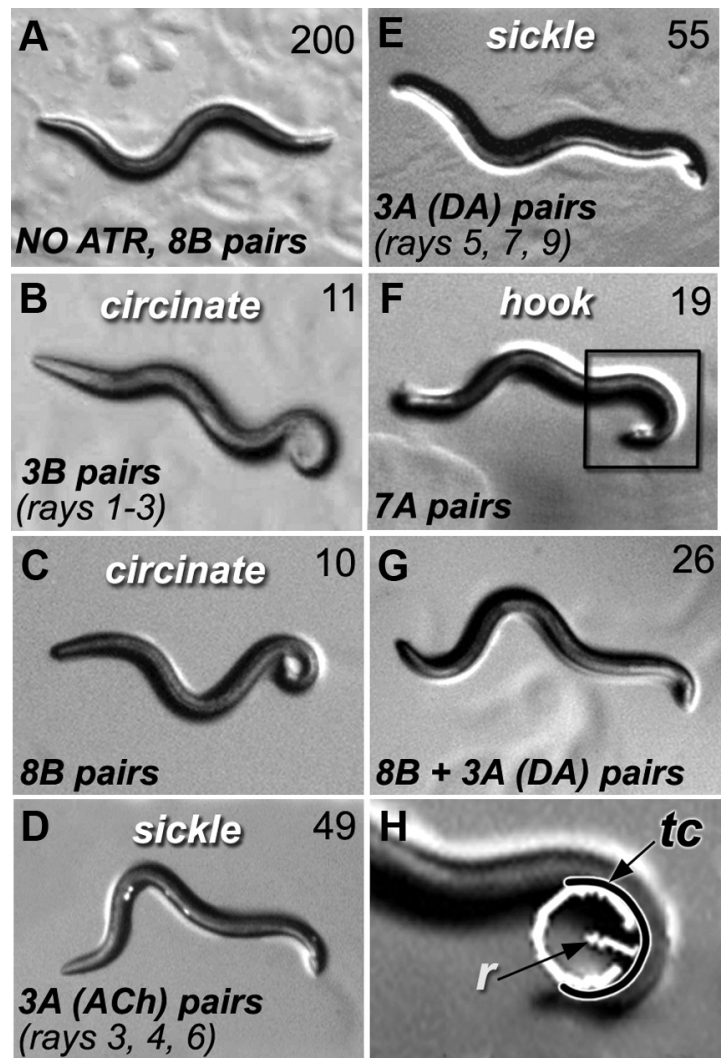

Figure 4. ChR2-mediated depolarization of A-or B-neurons induces different types of tail ventral curling. A-G, Images of ChR2-YFPtransgenic males showing tail posture generated after exposure to a 500 ms pulse of blue light. The number of ray neurons activated, and their cognate rays, is indicated. Tail-curve values are indicated in the top right of each image and were derived as shown in $\boldsymbol{H}$ and described in Materials and Methods. Except for $\boldsymbol{A}$, all males shown were grown in the presence of the ChR2 cofactor ATR. Circinate, sickle, and hook refer to the shape of the tail and posterior half of the animal. A circinate curl is coiled in a spiral with the tip innermost. $A$, NO ATR, 8B pairs: A ppkd-2::ChR2-YFP transgenic male grown in the absence of ChR2 cofactor ATR, expressing ChR2 in the B-neurons of eight of nine ray pairs. However, because of the absence of ATR, ChR2 is inactive. Under these conditions, transgenic males show no change in behavior after a blue light pulse, indicating that tail-curl response requires active channel and is not a nonspecific response to blue light. $\boldsymbol{B}, 3 \mathbf{B}$ pairs (rays 1 to 3 ): The tail posture of a male after activation of $B$-neurons in three ray pairs. The example shown is a ppkd-2::ChR2-YFP male expressing ChR2 only in the B-neurons of rays 1-3 (generated by eliminating $p p k d-2::$ ChR2-YFP-positive B-neurons of other rays through lasermediated ray cell rupturing). C, 8B pairs: A ppkd-2::ChR2-YFP transgenic male expressing ChR2 in the B-neurons of eight of nine ray pairs. D, 3A (ACh) pairs (rays 3, 4, 6): A ptba-9::ChR2-YFP male expressing ChR2 in the A-neurons of rays 3, 4, and 6, which are cholinergic (ACh). The tail makes a shallow ventral flexion, and the region between the tail-pivoting point and the midbody is relatively straight (in contrast to B-neuron-induced posture). E, 3A (DA) pairs (rays 5, 7, 9): A pdat-1::ChR2-YFP transgenic male expressing $C h R 2$ in the $A$-neurons of rays 5,7 , and 9, which are dopaminergic (DA). Posture is similar to that induced by A-neurons of rays 3, 4, and $6(\boldsymbol{D})$. $\boldsymbol{F}, 7$ A pairs: A ptba-9:: ChR2-YFP male expressing $C h R 2$ in the $A$-neurons of all rays except those of rays 5 and 8 . Note the deep ventral curl compared with the shallow tail curl produced by $A$-neurons subsets $(\boldsymbol{D}, \boldsymbol{E}) . \mathbf{G}, 8 \mathrm{~B}+3 \mathrm{~A}(\mathrm{DA})$ neuron pairs: A ppkd-2::ChR2-YFP; pdat-1::ChR2-YFP transgenic male expressing ChR2 in the neurons indicated. The tail posture generated more closely resembles an A-rather than a B-neuron-induced posture (compare with $\boldsymbol{C}, \boldsymbol{E}$ ). $\boldsymbol{H}$, Derivation of tail-curve values. Image corresponds to enlargement of boxed region in $\boldsymbol{F}$. The tail-curl value for a single male is determined from the frame showing the maximum degree of tail curling after the blue light pulse. A circle (white line) is drawn to fit the tail curl. The tail-curve value $=r \times$ [circle circumference/tc], where $r$ corresponds to the radius of curvature (white line, in pixels), and tc corresponds to the length of tail (in pixels) directly in contact with the circle (the black line).

B-neuron-induced response is relatively robust, with as few as three B-neuron pairs being capable of inducing a strong ventral curling response. Because B-neurons are important for inducing contact response (Fig. $2 \mathrm{~A}$ ), such a property would be predicted to 

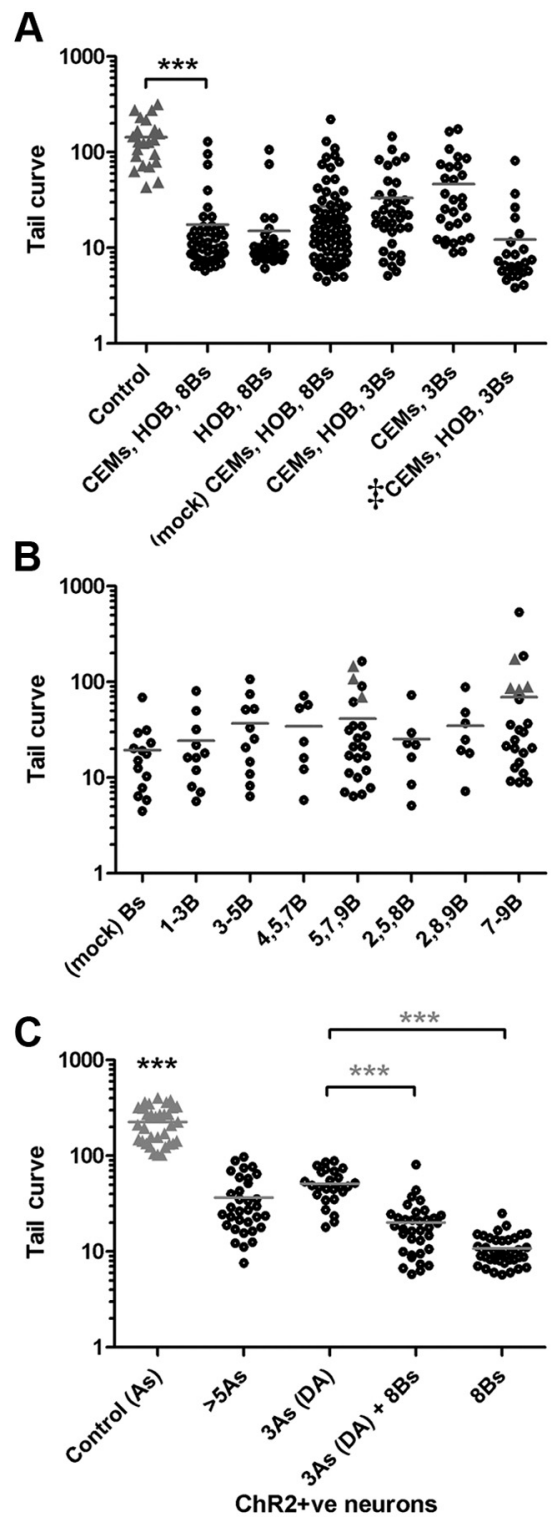

Figure 5. Quantification of A- and B-neuron-induced tail ventral curling. Quantification of tail curves generated by males after ChR2-mediated activation of the neurons indicated. Individual transgenic males expressing ChR2 in the neurons indicated on the $x$-axis were exposed to a $500 \mathrm{~ms}$ pulse of blue light. Tail-curve values ( $y$-axis, log scale) were calculated as described in Materials and Methods and shown in Figure $4 \mathrm{H}$. Each point corresponds to a single male: black circles (responders), tail ventral curl response with or without backing; gray triangles (nonresponders), no change in tail posture, forward locomotion uninterrupted. For non-responders, tail-curve values correspond to the curvature of the tail during normal forward sinusoidal locomotion at the time interval after the pulse a response would normally be observed. Results of nonparametric one-way ANOVA (Kruskal-Wallis test) are shown. Gray bars indicate mean. Significance is shown as ${ }^{* * *} p<0.001$. $A$, Tail curling in ppkd-2::ChR2-YFP transgenic males is attributable to stimulation of the ray B-neurons. ppkd-2::ChR2-YFP is expressed in the CEM and HOB neurons and in the B-neurons of eight pairs of rays (CEMs, HOB, 8Bs). ppkd-2::ChR2-YFP transgenic males grown in the absence of ATR (Control) show no change in behavior when pulsed with blue light, indicating that tail-curl response is not a nonspecific response to light exposure. For all other treatments shown, males were grown in the presence of ATR. ppkd-2::ChR2-YFP (CEMs, HOB, 8Bs) respond by adopting a tight ventral "circinate" curl with a mean tail-curve value of 20. Tail curling is attributable to $B$-neuron activation because elimination of the CEMs or $\mathrm{HOB}$ (HOB, $8 \mathrm{Bs}$ and $\mathrm{CEMs}, 3 \mathrm{BS}$, respectively) does not prevent tail curling. Males with only three $B$-neuron pairs (CEMs, $\mathrm{HOB}, 3 \mathrm{BS}$ ), generated by ablating the other six ray pairs, produce similar postures to males with all eight B-neuron pairs (mock CEMs, HOB, 8Bs). Males with three $B$-neuron pairs attributable to the presence of a lin-32 (atonal) mutant allele in the background ( $¥$ CEMs, HOB, 3Bs) (Zhao and Emmons, 1995) produce similar tail curls to males with three $B$-neuron pairs generated using the ray cell process rupturing technique. enhance the likelihood of tail apposition to the hermaphrodite after even limited ray contact.

ChR2-mediated A-neuron activation induced more open tail curls than the B-neurons (with A-neuron tail-curve values ranging from 10 to 100 ) (Fig. 4D-F) and was more sensitive to which and how many neurons were activated. When we activated subsets of A-neurons (either the cholinergic A-neurons of rays 3, 4, and 6 using ptba-9::ChR2-YFP or the dopaminergic A-neurons of rays 5, 7, and 9 using pdat-1::ChR2-YFP) males produced a relatively shallow ventral curl: the tail bent ventrally and the region from the midbody to the tail flexure point was often straight (see the "sickle"-like posture shown in Figs. 4D,E, 5C) (see Notes). This sickle posture resembles the scanning posture of males during mating. When we activated almost all A-neurons, $50 \%$ of males generated a deeper, hook-like ventral curl involving most of the posterior half of the animal, whereas the remaining males generated the sickle-like posture observed when A-neuron subsets were activated (Figs. 4F, 5C) (see Notes). The hook-like curl posture is remarkably similar to the turning posture observed during mating. The ability of A-neurons to induce such a posture when artificially activated is consistent with our finding that A-neurons are important for turning (Fig. 3C). Furthermore, our finding that the probability of such a posture is increased by inclusion of A-neurons from anterior rays in the activated set is consistent with data from our whole ray ablation experiments (Fig. $2 C$ ), in which males with anterior rays had a higher turn success rate than males with predominantly posterior rays.

Our A- and B-neuron targeted-ablation experiments suggest that both A- and B-neurons may be activated by initial contact. If so, what kind of posture is generated when both neuron types are artificially activated at the same time? To answer this, we simultaneously activated ChR2 in B-neurons (using the ppkd-2::ChR2-YFP transgene) and a small subset of A-neurons (using the pdat-1::ChR2-YFP transgene). Remarkably, $>50 \%$ of males produced a tail curl that more closely resembled an A-neuron-induced posture than the B-neuron circinate curl (compare Figs. 4C,E, G, 5C) (see Notes). Thus, the activation of even a few A-neuron pairs can significantly modify B-neuron-induced motor output.

\section{A- and B-neurons use different neurotransmitters to regulate tail posture}

A- and B-neuron populations can be divided into subtypes on the basis of their neurotransmitter fate. The A-neurons of rays 1-4 and ray 6 are cholinergic (data not shown), whereas those of rays

$\leftarrow$

ppkd-2::ChR2-YFP is not expressed in hermaphrodites. $\boldsymbol{B}$, ChR2-induced activation of different three B-neuron-pair combinations produces similar tail curls to eight B-neuron pairs (mock control). The numbers on $x$-axis correspond to the rays in which B-neurons were activated, for example, 1-3B corresponds to the B-neurons of rays $1-3$. Three-ray-pair combinations were generated by laser-mediated ray cell rupturing of specific rays. ChR2-positive CEMs and HOB cells are present in all treatments shown. C, Comparison of A-versus B-neuron induced tail-curl values. ptba-9::ChR2-YFP males expressing ChR2 in more than five pairs of ray neurons ( $>5 \mathrm{As}$ ) exhibit a deep ventral curl (hook) or a more open curl (sickle). 3As (DA) corresponds to pdat-1::ChR2-YFP males, which express ChR2 in the dopaminergic (DA) A-neurons of rays 5, 7, and 9. $3 \mathrm{~A}(\mathrm{DA})+8 \mathrm{~B}$ correspond to males with pdat-1::ChR2-YFP-positive A-neurons and all ppkd-2::ChR2-YFP-positive B-neurons. Coactivation of a small number of A-neurons modifies $B$-neuron output as indicated by the shift in tail-curl values compared with B-neurons alone. Control (As) corresponds to ptba-9::ChR2-YFP hermaphrodites grown in the presence of ATR Hermaphrodites, which lack ray neurons, exhibit no change in behavior when exposed to a pulse of blue light, indicating that tail curling in ptba-9::ChR2-YFP transgenic males is ray neuron dependent.pdat-1::ChR2-YFP hermaphrodites, which express the ChR2 in some of the same cells as ptba-9, namely the dopaminergic neurons of the core nervous system, also did not respond to blue light (data not shown). 


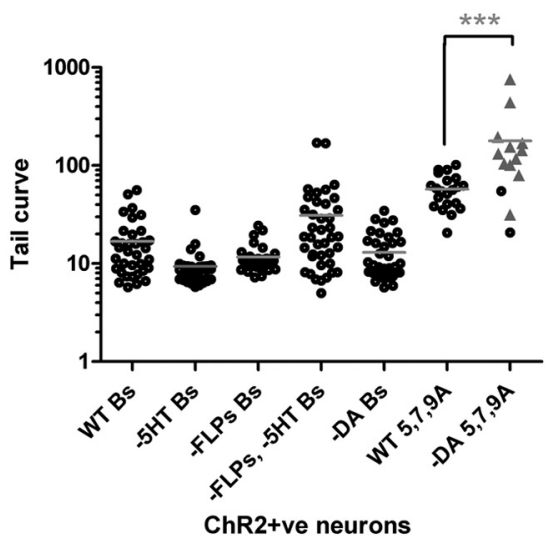

Figure 6. A- or B-neurons use different neurotransmitters to control tail posture. Tail-curve values for postures generated after ChR2-mediated activation of A- or B-neurons in wild-type and neurotransmitter-deficient mutant backgrounds. Each data point corresponds to a single animal. Black circles (responders), Tail ventral curl response with or without backing; gray triangles (non-responders), no change in tail posture, forward locomotion uninterrupted. For non-responders, tail-curve values correspond to the curvature of the tail during normal forward sinusoidal locomotion at the time interval after the pulse a response would normally be observed. Results of nonparametric one-way ANOVA (Kruskal-Wallis test) are shown. Gray bars indicate mean. Significance is shown as ${ }^{* * *} p<0.001$. B-neuron activation experiments were performed using the ppkd-2::ChR2-YFP transgenic males: wild type (WT Bs); serotonin-deficient tph-1 mutants (-5HT Bs); FMRF-amide peptide-deficient egl-3 mutants (-FLPs Bs); egl-3; tph-1 double mutants ( - FLPs $-5 H T$ Bs); and dopamine-deficient cat-2 mutants ( $-D A, B s)$. Dopaminergic A-neuron activation experiments were performed using the pdat-1::ChR2-YFP transgenic males. Shown are the tail curves generated after activation of these neurons in wild-type (WT 5, 7,9A) and cat-2 mutant $(-\mathrm{DA} 5,7,9 \mathrm{~A})$ males.

5, 7, and 9 are dopaminergic (Sulston and Horvitz, 1977). The B-neurons synthesize stereotyped combinations of FMRF-amide neuropeptides, encoded by different combinations of flp genes (for FMRF-like peptide), and a subset of these (those of rays 1, 3, and 9) additionally synthesize serotonin (Loer and Kenyon, 1993; Lints et al., 2004). To determine whether ray neurons use these various chemical messengers to induce tail ventral curling, we tested whether mutations that disrupt the synthesis of specific neurotransmitters or neuropeptides block ChR2-induced raydependent tail curling.

Mutations that specifically disrupt dopamine biosynthesis essentially abolished ChR2-induced tail ventral curling by the dopaminergic A-neurons. As described above, specific activation of dopaminergic A-neurons using the pdat-1::ChR2-YFP transgene induces a shallow ventral curling of the tail (Figs. 4F; 6, WT $5,7,9 \mathrm{~A})$. The gene cat-2 encodes the C. elegans tyrosine hydroxylase ortholog, and loss-of-function mutations in this gene reduce dopamine by $>95 \%$ (Sulston et al., 1975; Lints and Emmons, 1999). The presence of a cat-2 mutant allele in pdat-1::ChR2-YFP males rendered virtually all males nonresponsive to activation of the ChR2-expressing neurons (Fig. 6, -DA 5,7,9A); these males did not curl their tails when exposed to blue light and continued forward locomotion unperturbed. The inability of cat-2 males to respond was not attributable to a general decrease in nervous system responsiveness: cat-2 loss of function did not significantly affect induction of tail curling by ChR2-expressing B-neurons (Fig. 6, -DA Bs). Other A-neurons are cholinergic, but we were unable to test whether these neurons use cholinergic transmission to induce tail ventral curling because acetylcholine is made by many neurons in the nervous system and mutants are severely uncoordinated (for review, see Rand, 2007).

The B-neuron neurotransmitters serotonin and FMRF-amide neuropeptides are not essential for B-neuron-induced tail curl- ing. Males deficient in serotonin synthesis [tph-1 mutants (Sze et al., 2000)] or FMRF-amide peptide processing [egl-3 mutants (Kass et al., 2001)] generated a tail curl when ChR2-positive B-neurons were exposed to blue light (Fig. 6). tph-1; egl-3 double mutants also responded to artificial B-neuron activation by curling their tails, although less robustly than wild-type males. This suggests that serotonin and FMRF-amide peptides may have partially redundant modulatory roles in mating posture control. The mode of transmission used by B-neurons to induce the tail-curling motor response is therefore still an open question.

\section{Many male-specific ray neuron targets are not essential for ventral curling}

Ray neurons form connections with a large number of cells in the male posterior, both male-specific and gender-common or "core" cell types (Male Wiring Project, Albert Einstein College of Medicine, http://worms.aecom.yu.edu/PHP/male_wiring_project. php). The core cell targets include the ventral nerve cord motor neurons and body wall muscles that promote sinusoidal wave propagation during normal locomotion and the command interneurons that influence its direction (Chalfie et al., 1985; de Bono and Maricq, 2005; Guo et al., 2009). Among the malespecific cells are 21 cells classed as interneurons and/or motor neurons that connect ray neurons either directly or indirectly core cell types. Most of these neurons are located in the pre-anal ganglion to which the axons of all ray neurons project. In addition, 35 male-specific muscles supplement the musculature of the ventral posterior body wall (Sulston and Horvitz, 1977; Sulston et al., 1980) (Male Wiring Project, Albert Einstein College of Medicine, http://worms.aecom.yu.edu/PHP/male_wiring_project. php). This connectivity raises the question, to what extent does ray control of body posture depend on male-specific versus core efferent targets? To begin to address this issue, we took advantage of mutations in the Hox genes mab-5 (Antennapedia homolog) and lin-39 (Sex-combs-reduced homolog), which block the generation of different subsets of male-specific cells (Kenyon, 1986; Clark et al., 1993; Salser et al., 1993; Wang et al., 1993). We introduced the B-neuron $p p k d-2:: C h R 2-Y F P$ transgene into these backgrounds and asked whether mutant males could curl their tails when B-neurons were stimulated with blue light. We chose the B-neuron transgene because rays 1-6 are not generated in mab-5 mutants, but experiments described above indicate that $B$-neuron-induced tail curling is relatively insensitive to the number of B-neurons stimulated (Fig. $5 A, B$ ). In addition to missing rays $1-6$, mab-5 mutants fail to generate all 41 malespecific muscles and 11 of 15 male-specific cells that contribute to the pre-anal ganglion and posterior ventral nerve cord, namely neurons PDC, PVV, PVY, PVZ, CP7-CP9, PGA, and neuron-like cells CA7-CA9 (Kenyon, 1986). In wild-type males, except for CA7-CA9, all mab-5-dependent cells have direct inputs from the B-neurons of rays 7-9 (Male Wiring Project, Albert Einstein College of Medicine, http://worms.aecom.yu.edu/PHP/male wiring_project.php). PDC, PVV, PVY, and PVZ may function as motor neurons in some circuits but as interneurons in others based on the identities of their various postsynaptic partners; hence, we refer to neurons of this type as interneurons/motor neurons. Based on their connectivity, CP7-CP9 may function purely as interneurons (Male Wiring Project, Albert Einstein College of Medicine, http://worms.aecom.yu.edu/PHP/male wiring_project.php). Surprisingly, the absence of this large contingent of male-specific cells in mab-5 null mutant males does not completely block B-neuron-induced tail curling. mab-5 males can curl their tails, although typically less tightly or less smoothly 


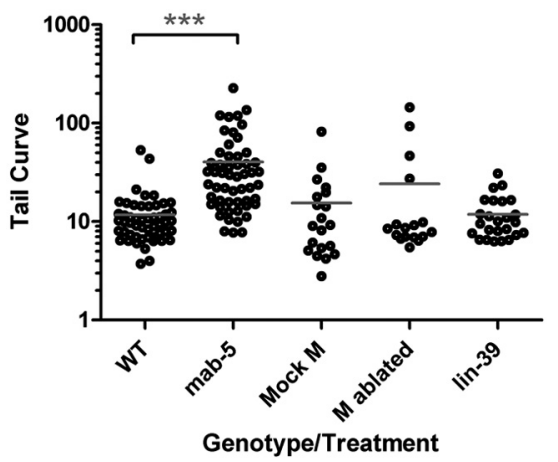

Figure 7. Many male-specific ray neuron cellular targets are not essential for ray-induced tail ventral curling. Tail-curve values generated after activation of B-neurons in males of the genotype or treatment shown. Males in all categories carry the ppkd-2::ChR2-YFP transgene. WT corresponds to genetically wild-type males in which all $p p k d 2$-expressing cells are present (CEMs, HOB, and 8 pairs of ray B-neurons). mab-5 males express ChR2-YFP in the CEMs and HOB but only three B-neuron pairs, the B-neurons of rays $7-9$. Although significantly less robust than wild-type males, mab-5 males can curl their tails despite the absence of a significant number of male-specific cell types. Mock M corresponds to mock control males for M ablations. $M$ ablated corresponds to males lacking all male-specific muscles as a result of laser ablation of their common precursor M. lin-39 males lack the six CP and six CA motor neurons of the ventral nerve cord. Results of nonparametric one-way ANOVA (Kruskal-Wallis test) are shown. Gray bars indicate mean. Significance is shown as ${ }^{* *} p<0.001$.

than control males (Fig. 7) (see Notes). The less robust ventral curling displayed by mab-5 males could be attributable to the absence of male-specific muscles, neurons, or both. To determine how much male-specific muscles contribute to ventral curling, we eliminated these cells by ablating their common precursor, $\mathrm{M}$, in otherwise wild-type males carrying the B-neuron $p p k d 2:: C h R 2-Y F P$ transgene. $\mathrm{M}$-ablated males exhibited variable tail curling, much like mab-5 males (Fig. 7) (see Notes). This suggests that the main cause of variable tail curling in mab-5 males is the absence of male-specific muscles rather than the absence of the interneurons or motor neurons.

In lin-39 mutant males, two lineally related classes of malespecific ventral cord motor neurons are not specified: the serotonergic neurons CP1-CP6 and their sister cells CA1-CA6 (Clark et al., 1993; Loer and Kenyon, 1993). Some of these neurons directly innervate the gonad and are necessary for ejaculation (Male Wiring Project, Albert Einstein College of Medicine, http://worms. aecom.yu.edu/PHP/male_wiring_project.php) (Schindelman et al., 2006). In addition, a number of CP/CA neurons synapse onto ventral body wall muscles, suggesting that they may regulate ventral curling. However, we find that lin-39 males produce tail curls similar to wild type when ChR2-expressing B-neurons are stimulated. Thus, B-neurons do not require CP/CA1-6 motor neurons to induce contraction of ventral muscles and may use alternative pathways to achieve this effect.

Together, these data show that eliminating many malespecific neurons that are direct ray neuron targets or malespecific muscles that are appropriately located to affect ventral bending does not completely block B-neuron-induced tail curling. Indeed, some M-ablated males produce a near wild-type tail curl, indicating that the system is capable of compensatory activity that can overcome the loss of male-specific muscles. The fact that $\mathrm{M}$-ablated males produce variable tail curls suggests that posture control is distributed across male-specific and core muscle targets (as suggested by Whittaker and Sternberg, 2009). The male-specific muscles and interneurons/motor neurons may hone the finer details of postural control that enhance the efficiency of mate apposition sub-behaviors.

\section{Discussion}

C. elegans male mating behavior provides an opportunity to dissect the most complex behavior exhibited by the anatomically best-understood metazoan nervous system. Coupled with the genetic amenability of this system, the single-cell resolution analysis of ray neuron function also provides a facile model for tackling the fundamental neuroscience question of how robustness in neural circuit activity and output is supported at the cellular and molecular level.

In this study, we have investigated the neural basis of one aspect of male C. elegans mating behavior, mate apposition behavior. This complex sensorimotor behavior is triggered by hermaphrodite contact and terminated by location of the vulva. Apposition behavior can be divided into three sub-behaviors that all involve backward locomotion and pressing of the male tail against the hermaphrodite surface: contact response, scanning, and turning. We show that elimination of all male sensory rays renders males essentially nonresponsive to contact, indicating that the rays are essential for inducing tail placement and prompting backward locomotion. We find that there is considerable functional overlap among the nine bilateral pairs of rays in contact response (Fig. 8). As few as three ray pairs can mediate contact response, and all subsets tested were essentially equivalent. Males with three-ray-pair combinations could also mediate scanning and turning, but here efficiency was more variable across subsets. Males with anterior ray sets (rays 1-3 and rays 3-5) were more efficient in scanning and turning than males with predominantly posterior rays, a result that may reflect differences in neurotransmitter fate and/or connectivity among the A-neurons of anterior and posterior rays.

Functional overlap among rays stems from all rays possessing the same sensory neuron types with similar functional properties. When artificially activated with $\mathrm{ChR} 2$, the B-neurons and, to a lesser extent, A-neurons of different rays induce similar motor output. Functional difference among rays is likely attributable to differences in connectivity and neurotransmitter fates. The A-neurons of rays 5, 7, and 9 are dopaminergic and require dopamine to induce tail-curling response when artificially stimulated using ChR2. The A-neurons of rays $1-4$ and ray 6 are cholinergic, and, although we have not demonstrated that these neurons use acetylcholine to induce postural changes, other studies implicate acetylcholine in tail posture control (Whittaker and Sternberg, 2009). Surprisingly, we find that the known neurotransmitters of the B-neurons, serotonin and FMRF-amides, are not essential for B-neuron-induced posture. Their role appears to be modulatory because their absence reduces the response robustness, but not induction, of B-neuron-induced tail curling. In the hermaphrodite, serotonin and FMRF-amides have complex neuromodulatory effects on several worm motor behaviors, including egg laying and locomotion (Nelson et al., 1998; Rogers et al., 2006; Hapiak et al., 2009).

Specific elimination of A- or B-neurons reveals that these neuron types do not contribute equally to all apposition subbehaviors (summarized in Fig. 8). Both A- and B- neurons are required for contact response. However, artificial stimulation of each type suggests that A- and B-neurons may make different contributions to postural control. When artificially activated, A-neuron subsets produce a shallow, open curl, whereas B-neurons induce a robust tight curl. Curiously, A- and B-neuron coactivation can produce a mostly A-neuron-like posture, indicating that the output of even a small number of A-neurons can significantly impact B-neuron-induced postures. A-neuron-induced postures 
would presumably expose the hook and post-cloacal sensilla more effectively to the hermaphrodite surface than the circinate Bneuron-induced posture. Conversely, Bneuron activation might enhance the strength and likelihood of initial responsiveness, and the muscle groups they activate may confer greater downward tail force onto the hermaphrodite surface, securing the necessary contact. Scanning was unaffected by the absence of B-neurons but strongly affected when A-neuron number was reduced, indicating that continued activity of A-neurons is critical for scanning posture control. Turning was also specifically affected by a reduction in the A-neuron population. Consistent with this, ChR2 activation of all A-neurons induces turninglike postures. Because activation of all A-neurons produces both scanning- and turning-like postures, factors other than simply the number of neurons stimulated must shift the balance toward one posture or the other. Sensitive bistability in the output of A-neurons may represent a tipping point on which unknown hermaphrodite cues act to indicate that the hermaphrodite head or tail is close by. Although B-neurons are dispensable for scanning or turning, it is possible they may contribute to these sensorimotor behaviors in more subtle ways, given their convergent connectivity on many Aneuron cellular targets.

More complex nervous systems use large fields of sensory cells to extract salient information from the environment and determine the whereabouts and actions of potential conspecific mates, competitors, and predators. Large fields of cells with differing sensitivities and tuned to different stimulus features confer robust response properties (Lewis, 1999; Kawamura and Tachibanaki, 2008; Schwander et al., 2010). The ray sensilla cover a significant region of the male tail, thus providing a large surface area for contact with the hermaphrodite. Our results reveal a striking degree of robustness in ray function, such that in the face of significant experimental perturbation males are still able to exercise fine control over tail posture and locomotion in response to moment-to-moment changes in hermaphrodite contours (or other surface cues).

Our data suggests that ray sensorimotor circuit robustness is not solely encoded at the sensory neuron level but may feature in processing at multiple levels of the circuit. If robustness were limited to the sensory neurons, we would predict that elimination of downstream ray targets would completely block ray-induced posture. However, we find that this is not the case. In mab-5 Hox gene mutants, a significant number of male-specific interneurons/motor neurons and muscles are absent, and yet we find that mutant males can still produce ventral tail curling when Bneurons are artificially activated with ChR2. This suggests that processing downstream of the rays is distributed across multiple interneuron, motor neuron, and muscle targets, as the ray connectivity data suggest. A distributed processing model would also explain how ray neurons could rapidly effect subtle postural changes, some involving the entire male posterior half, during the course of the search. Ray neuron targets include both malespecific and core system cell types, and our analyses indicate that both cell classes contribute to ray-neuron-dependent posture

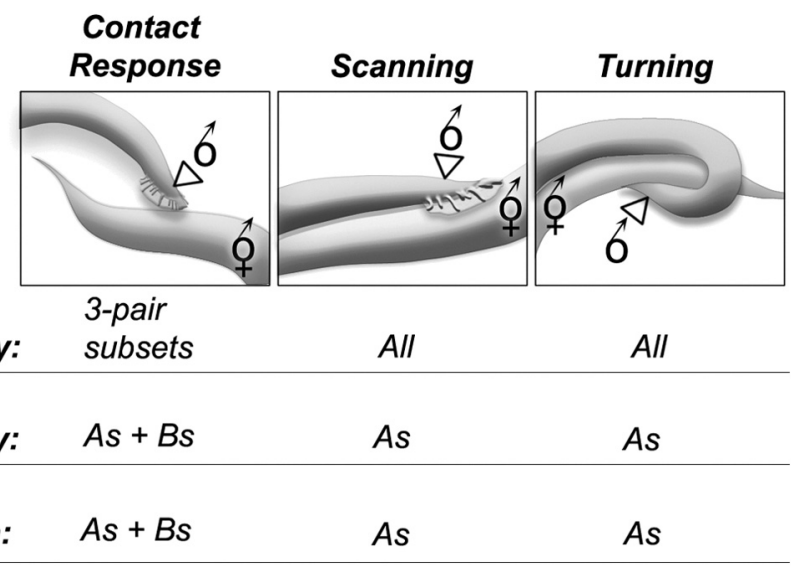

Figure 8. Summary of ray functional analyses. Top row is a diagram showing examples of male tail posture during contact response, scanning, and turning. Only the posterior half of the male is shown with the male tail indicated by the arrowhead. Indicated below are the rays and ray neuron types required for each sub-behavior and which ray neurons generate a posture similar 列

control. Other studies have shown that the fluidity of rayregulated backward locomotion during mating is modulated by core sensory neurons (T. Liu et al., 2007), indicating that, like posture control, locomotion is controlled by both core and malespecific system components. In the context of posture control, we find that some mab-5 and $\mathrm{M}$-ablated males could generate near wild-type tail curls, revealing that activity of core muscles and remaining neurons is sometimes sufficient to compensate for network cell loss. Similar core-nervous system-dependent compensation has been described in the sensory circuits controlling another behavior critical to C. elegans mating success, namely mate attraction (White et al., 2007). In vertebrates, the developing mammalian retina and hippocampus provide striking examples of such homeostatic compensation, which similarly involves the activation of parallel or latent circuits (Blankenship and Feller, 2010). Dramatic functional robustness and compensatory circuit dynamics have also long been recognized in the operation of central pattern generators supporting different locomotor modes in intact and manipulated animals (von Holst, 1937). From the C. elegans nervous system wiring diagram, we can identify potential compensatory or parallel pathways with single-cell resolution and assess their contribution. For example, in mab-5 males, there are a number of alternative pathways that could compensate for cell losses. The ray neurons connect directly to both core muscles and to the core ventral cord neurons that innervate them. Core neuron PDB has identical connectivity to male-specific PDC interneuron/motor neuron (absent in mab5). There is no obvious core nervous system counterpart for the CP7-CP9 interneurons (absent in mab-5), but male-specific EF interneurons could provide an alternative because these two groups of cells have common presynaptic and postsynaptic partners.

The generation of such resilient cellular networks is likely to be driven by natural selection for mating success during C. elegans evolution. With knowledge of the C. elegans cell lineage and connectivity, we can speculate on the structure of the primordial ray circuit on which the extant circuitry is based. Studies from $C$. elegans, Drosophila, and mouse reveal that sexual behaviors can 
be the product of gender-specific modulation of circuitry present in both sexes (Kimchi et al., 2007; Lee and Portman, 2007; White et al., 2007; Clyne and Miesenböck, 2008). Given this, direct connections between rays and core cells may represent the foundation on which the ray sensorimotor circuits are based. Many of the male-specific interneuron/motor neurons, such as PDC, PVX, PVZ, and PVV, are generated from iterated lineages that also produce the core locomotor motor neurons of the ventral nerve cord. Thus, these male-specific motor neurons may correspond to derived ventral nerve cord motor neurons that provide additional control of muscle contraction patterns in the male posterior and thus contribute to the functional resilience of the network.

\section{Notes}

Supplemental Movies 1-11 are available at http://www.bio.tamu.edu/ users/rlints/supplementals.html. This material has not been peer reviewed.

\section{References}

Bargmann CI (2006) Chemosensation in C. elegans (The C. elegans Research Community, ed). Available at WormBook, http://www.wormbook.org, doi/10.1895/wormbook.1.123.1.

Bargmann CI, Avery L (1995) Laser killing of cells in Caenorhabditis elegans. Methods Cell Biol 48:225-250.

Bargmann CI, Horvitz HR (1991) Chemosensory neurons with overlapping functions direct chemotaxis to multiple chemicals in C. elegans. Neuron 7:729-742.

Barker DM (1994) Copulatory plugs and paternity assurance in the nematode, Caenorhabditis elegans. Anim Behav 48:147-156.

Barr MM, Sternberg PW (1999) A polycystic kidney-disease gene homologue required for male mating behaviour in C. elegans. Nature 401:386-389.

Barr MM, DeModena J, Braun D, Nguyen CQ, Hall DH, Sternberg PW (2001) The Caenorhabditis elegans autosomal dominant polycystic kidney disease gene homologs $l o v-1$ and $p k d-2$ act in the same pathway. Curr Biol 11:1341-1346.

Barrios A, Nurrish S, Emmons SW (2008) Sensory regulation of C. elegans male mate-searching behavior. Curr Biol 18:1865-1871.

Biron D, Wasserman S, Thomas JH, Samuel AD, Sengupta P (2008) An olfactory neuron responds stochastically to temperature and modulates Caenorhabditis elegans thermotactic behavior. Proc Natl Acad Sci U S A 105:11002-11007.

Blankenship AG, Feller MB (2010) Mechanisms underlying spontaneous patterned activity in developing neural circuits. Nat Rev Neurosci 11:18-29.

Brenner S (1974) The genetics of Caenorhabditis elegans. Genetics 77:71-94.

Chalfie M, Sulston J (1981) Developmental genetics of the mechanosensory neurons of Caenorhabditis elegans. Dev Biol 82:358-370.

Chalfie M, Sulston JE, White JG, Southgate E, Thomson JN, Brenner S (1985) The neural circuit for touch sensitivity in Caenorhabditis elegans. J Neurosci 5:956-964.

Chelur DS, Chalfie M (2007) Targeted cell killing by reconstituted caspases. Proc Natl Acad Sci U S A 104:2283-2288.

Chelur DS, Ernstrom GG, Goodman MB, Yao CA, Chen L, O’ Hagan R, Chalfie M (2002) The mechanosensory protein MEC-6 is a subunit of the C. elegans touch-cell degenerin channel. Nature 420:669-673.

Christensen AP, Corey DP (2007) TRP channels in mechanosensation: direct or indirect activation? Nat Rev Neurosci 8:510-521.

Clark SG, Chisholm AD, Horvitz HR (1993) Control of cell fates in the central body region of C. elegans by the homeobox gene lin-39. Cell 74:43-55.

Clyne JD, Miesenböck G (2008) Sex-specific control and tuning of the pattern generator for courtship song in Drosophila. Cell 133:354-363.

Collet J, Spike CA, Lundquist EA, Shaw JE, Herman RK (1998) Analysis of osm-6, a gene that affects sensory cilium structure and sensory neuron function in Caenorhabditis elegans. Genetics 148:187-200.

Coste B, Mathur J, Schmidt M, Earley TJ, Ranade S, Petrus MJ, Dubin AE, Patapoutian A (2010) Piezo1 and Piezo2 are essential components of distinct nechanically activated cation channels. Science 330:55-60.

de Bono M, Maricq AV (2005) Neuronal substrates of complex behaviors in C. elegans. Annu Rev Neurosci 28:451-501.
Garcia LR, LeBoeuf B, Koo P (2007) Diversity in mating behavior of hermaphroditic and male-female Caenorhabditis nematodes. Genetics 175:1761-1771.

Garrity PA, Goodman MB, Samuel AD, Sengupta P (2010) Running hot and cold: Behavioral strategies, neural circuits, and the molecular machinery for thermotaxis in C. elegans and Drosophila. Genes Dev 24:2365-2382.

Goodman MB (2006) Mechanosensation (The C. elegans Research Community, ed). Available at WormBook, http://www.wormbook.org, doi/10.1895/wormbook.1.62.1.

Granato M, Schnabel H, Schnabel R (1994) Genesis of an organ: molecular analysis of the pha-1 gene. Development 120:3005-3017.

Guo ZV, Hart AC, Ramanathan S (2009) Optical interrogation of neural circuits in Caenorhabditis elegans. Nat Methods 6:891-896.

Hapiak VM, Hobson RJ, Hughes L, Smith K, Harris G, Condon C, Komuniecki P, Komuniecki RW (2009) Dual excitatory and inhibitory serotonergic inputs modulate egg laying in Caenorhabditis elegans. Genetics 181:153-163.

Hills T, Brockie PJ, Maricq AV (2004) Dopamine and glutamate control area-restricted search behavior in Caenorhabditis elegans. J Neurosci 24:1217-1225.

Hodgkin J, Horvitz HR, Brenner S (1979) Nondisjunction mutants of the nematode Caenorhabditis elegans. Genetics 91:67-94.

Hurd DD, Miller RM, Núñez L, Portman DS (2010) Specific $\alpha$ - and $\beta$-tubulin isotypes optimize the functions of sensory cilia in Caenorhabditis elegans. Genetics 185:883-896.

Kang L, Gao J, Schafer WR, Xie Z, Xu XZ (2010) C. elegans TRP family protein TRP- 4 is a pore-forming subunit of a native mechanotransduction channel. Neuron 67:381-391.

Kass J, Jacob TC, Kim P, Kaplan JM (2001) The EGL-3 proprotein convertase regulates mechanosensory responses of Caenorhabditis elegans. J Neurosci 21:9265-9272.

Kawamura S, Tachibanaki S (2008) Rod and cone photoreceptors: molecular basis of the difference in their physiology. Comp Biochem Physiol A Mol Integr Physiol 150:369-377.

Kenyon C (1986) A gene involved in the development of the posterior body region of C. elegans. Cell 46:477-487.

Kimchi T, Xu J, Dulac C (2007) A functional circuit underlying male sexual behaviour in the female mouse brain. Nature 448:1009-1014.

Kleemann G, Basolo A (2007) Facultative decrease in mating resistance in hermaphroditic Caenorhabditis elegans with self-sperm depletion. Anim Behav 74:1339-1347.

Knobel KM, Peden EM, Barr MM (2008) Distinct protein domains regulate ciliary targeting and function of C. elegans PKD-2. Exp Cell Res 314:825-833.

Lee K, Portman DS (2007) Neural sex modifies the function of a C. elegans sensory circuit. Curr Biol 17:1858-1863.

Lewis JE (1999) Sensory processing and the network mechanisms for reading neuronal population codes. J Comp Physiol A Neuroethol Sens Neural Behav Physiol 185:373-378.

Li W, Feng Z, Sternberg PW, Xu XZ (2006) A C. elegans stretch receptor neuron revealed by a mechanosensitive TRP channel homologue. Nature 440:684-687.

Lints R, Emmons SW (1999) Patterning of dopaminergic neurotransmitter identity among Caenorhabditis elegans ray sensory neurons by a TGFbeta family signaling pathway and a Hox gene. Development 126:5819-5831.

Lints R, Jia L, Kim K, Li C, Emmons SW (2004) Axial patterning of C. elegans male sensilla identities by selector genes. Dev Biol 269:137-151.

Liu KS, Sternberg PW (1995) Sensory regulation of male mating behavior in Caenorhabditis elegans. Neuron 14:79-89.

Liu T, Kim K, Li C, Barr MM (2007) FMRFamide-like neuropeptides and mechanosensory touch receptor neurons regulate male sexual turning behavior in Caenorhabditis elegans. J Neurosci 27:7174-7182.

Liu Y, LeBoeuf B, Garcia LR (2007) G $\alpha_{\mathrm{q}}$-coupled muscarinic acetylcholine receptors enhance nicotinic acetylcholine receptor signaling in Caenorhabditis elegans mating behavior. J Neurosci 27:1411-1421.

Loer CM, Kenyon CJ (1993) Serotonin-deficient mutants and male mating behavior in the nematode Caenorhabditis elegans. J Neurosci 13:54075417.

Mello C, Fire A (1995) DNA transformation. Methods Cell Biol 48:451-482.

Miller RM, Portman DS (2010) A latent capacity of the C. elegans polycys- 
tins to disrupt sensory transduction is repressed by the single-pass ciliary membrane protein CWP-5. Dis Model Mech 3:441-450.

Nagel G, Brauner M, Liewald JF, Adeishvili N, Bamberg E, Gottschalk A (2005) Light activation of Channelrhodopsin-2 in excitable cells of Caenorhabditis elegans triggers rapid behavioral responses. Curr Biol 15:2279-2284.

Nelson LS, Rosoff ML, Li C (1998) Disruption of a neuropeptide gene, flp-1, causes multiple behavioral defects in Caenorhabditis elegans. Science 281:1686-1690.

Rand JB (2007) Acetylcholine (The C. elegans Research Community, ed). Available at WormBook, http://www.wormbook.org, doi/10.1895/wormbook.1.131.1.

Rogers C, Persson A, Cheung B, de Bono M (2006) Behavioral motifs and neural pathways coordinating $\mathrm{O} 2$ responses and aggregation in C. elegans. Curr Biol 16:649-659.

Saifee O, Wei L, Nonet ML (1998) The Caenorhabditis elegans unc-64 locus encodes a syntaxin that interacts genetically with synaptobrevin. Mol Biol Cell 9:1235-1252.

Salser SJ, Loer CM, Kenyon C (1993) Multiple HOM-C gene interactions specify cell fates in the nematode central nervous system. Genes Dev $7: 1714-1724$.

Schindelman G, Whittaker AJ, Thum JY, Gharib S, Sternberg PW (2006) Initiation of male sperm-transfer behavior in Caenorhabditis elegans requires input from the ventral nerve cord. BMC Biol 4:26.

Schwander M, Kachar B, Müller U (2010) The cell biology of hearing. J Cell Biol 190:9-20.

Schwartz HT, Horvitz HR (2007) The C. elegans protein CEH-30 protects male-specific neurons from apoptosis independently of the Bcl-2 homolog CED-9. Genes Dev 21:3181-3194.
Sidi S, Friedrich RW, Nicolson T (2003) NompC TRP channel required for vertebrate sensory hair cell mechanotransduction. Science 301:96-99.

Sulston JE, Horvitz HR (1977) Post-embryonic cell lineages of the nematode, Caenorhabditis elegans. Dev Biol 56:110-156.

Sulston J, Dew M, Brenner S (1975) Dopaminergic neurons in the nematode Caenorhabditis elegans. J Comp Neurol 163:215-226.

Sulston JE, Albertson DG, Thomson JN (1980) The Caenorhabditis elegans male: Postembryonic development of nongonadal structures. Dev Biol 78:542-576.

Sze JY, Victor M, Loer C, Shi Y, Ruvkun G (2000) Food and metabolic signalling defects in a Caenorhabditis elegans serotonin-synthesis mutant. Nature 403:560-564.

von Holst E (1937) On the nature of order in the central nervous system. In: The organization of action: a new synthesis (Gallistel CR, ed), pp 81-110. Hillsdale, NJ: Erlbaum.

Walker RG, Willingham AT, Zuker CS (2000) A Drosophila mechanosensory transduction channel. Science 287:2229-2234.

Wang BB, Müller-Immergluck MM, Austin J, Robinson NT, Chisholm A, Kenyon C (1993) A homeotic gene cluster patterns the anteroposterior body axis of C. elegans. Cell 74:29-42.

White JQ, Nicholas TJ, Gritton J, Truong L, Davidson ER, Jorgensen EM (2007) The sensory circuitry for sexual attraction in C. elegans males. Curr Biol 17:1847-1857.

Whittaker AJ, Sternberg PW (2009) Coordination of opposing sex-specific and core muscle groups regulates male tail posture during Caenorhabditis elegans male mating behavior. BMC Biol 7:33-48.

Zhao C, Emmons SW (1995) A transcription factor controlling development of peripheral sense organs in C. elegans. Nature 373:74-78. 